\title{
Diagnóstico y tratamiento de la retinopatía diabética y edema macular diabético: guía de práctica clínica del Seguro Social de Salud del Perú (EsSalud)

\author{
Diagnosis and treatment of diabetic retinopathy and diabetic macular edema: clinical practice
} guidelines of the Peruvian Health Social Security (EsSalud)
}

Javier Héctor Cáceres-del-Carpio ${ }^{1,2,3}$, Renzo Cañote Flores ${ }^{4}$, José Montes-Alvis ${ }^{2}$, Kevin Pacheco-Barrios ${ }^{2}$, Doris Quiroz-Cerna ${ }^{5}$, Vanesa Cinthia Luján-Donayre ${ }^{5}$, Guillermo Reátegui-Escalante ${ }^{6}$, Robinson Richard Escobedo-Arriola ${ }^{6}$, Pedro Manuel García-Rodríguez ${ }^{6}$, Enrique Chacón-Rosello ${ }^{7}, J^{2}$. Yolanda ChahuaTorres $^{8}$, Harvy Alberto Honorio-Morales ${ }^{9}$, Antonio Martín Touzett-Valera ${ }^{6}$, Héctor Eduardo ShimabukuYsa ${ }^{9}$, Lesly Chávez-Rimache ${ }^{2}$, Alvaro Taype-Rondan², Raúl Timaná-Ruiz

${ }^{1}$ Hospital Nacional Edgardo Rebagliati Martins, Seguro Social de Salud (EsSalud). Lima, Perú.

${ }^{2}$ Instituto de Evaluación de Tecnologías en Salud e Investigación, Seguro Social de Salud (EsSalud). Lima, Perú.

${ }^{3}$ Facultad de Medicina Humana Manuel Huamán Guerrero, Universidad Ricardo Palma. Lima, Perú.

${ }^{4}$ Instituto de Ojos Oftamosalud. Lima, Perú.

${ }^{5}$ Hospital Nacional Guillermo Almenara Irigoyen, Seguro Social de Salud (EsSalud). Lima, Perú.

${ }^{6}$ Instituto Nacional de Oftalmologia, Ministerio de Salud del Perú. Lima, Perú.

${ }^{7}$ Hospital Nacional Hipólito Unanue, Ministerio de Salud del Perú. Lima, Perú.

${ }^{8}$ Hospital Nacional Dos de Mayo, Ministerio de Salud del Perú. Lima, Perú.

${ }^{9}$ Estrategia Sanitaria Nacional de Salud Ocular y Prevención de la Ceguera, Ministerio de Salud del Perú. Lima, Perú.

Códigos ORCID: Javier Héctor Cáceres-del-Carpio: https://orcid.org/0000-0001-5673-5709; Kevin Pacheco-Barrios: https://orcid.org/0000-0002-7166-2816; Harvy Alberto HonorioMorales: https://orcid.org/0000-0003-2661-9971; Lesly Chávez-Rimache: https://orcid.org/0000-0001-9987-7475; Álvaro Taype-Rondan: https://orcid.org/0000-0001-8758-0463

Correspondencia:

Raúl Timaná Ruiz

raul.timana@essalud.gob.pe

Recibido: 30 de enero 2020

Aprobado: 29 de febrero 2020

Publicación en línea: 30 de marzo de 2020

Conflictos de interés: Los responsables de la elaboración del presente documento declaran no tener ningún conflicto de interés financiero o no financiero, con relación a los temas descritos.

Fuente de financiamiento: Este documento técnico ha sido financiado por el Instituto de Evaluación de Tecnologías en Salud e Investigación (IETSI), EsSalud, Perú.

Contribuciones de autoría: Todos los autores participaron en la realización de la guía. Kevin Pacheco-Barrios y José MontesAlvis se encargaron de las búsquedas sistemáticas, la evaluación de calidad de los estudios y la evaluación de la certeza de la evidencia para cada pregunta. Lesly Chávez-Rimache redactó la primera versión del artículo. Todos los autores participaron en la discusión de los estudios encontrados y la formulación de las recomendaciones y puntos de buenas prácticas clínicas. Todos los autores revisaron y aprobaron la versión final del artículo.

Citar como: Cáceres-del-Carpio JH, Cañote R, Montes-Alvis J, PachecoBarrios K, Quiroz-Cema D, Lujan-Donayre VC et al. Diagnóstico y tratamiento de la retinopatía diabética y edema macular diabético: guía de práctica clínica del Seguro Social de Salud del Perú (EsSalud). An Fac med. 2020;81(1): 113122. DOl: https://doi.org/10.15381/anales. v81i1.17792
An Fac med. 2020; 81(1):113-22. / DOI: https://doi.org/10.15381/anales.v81i1.17792

\section{Resumen}

Introducción. El presente artículo resume la guía de práctica clínica (GPC) para el diagnóstico y tratamiento de la retinopatía diabética y el edema macular diabético en el Seguro Social de Salud del Perú (EsSalud). Objetivo. Proveer recomendaciones clínicas basadas en evidencia para el diagnóstico y tratamiento de la retinopatía diabética y el edema macular diabético en EsSalud. Métodos. Se conformó un grupo elaborador de la guia (GEG) que incluyó médicos especialistas y metodólogos. EI GEG formuló 4 preguntas clínicas a ser respondidas por la presente GPC. Para cada una de estas preguntas se realizó búsquedas de revisiones sistemáticas y de estudios primarios (cuando se consideró pertinente) en PubMed durante el 2018. Se seleccionó la evidencia para responder cada una de las preguntas clínicas planteadas. La certeza de la evidencia fue evaluada usando la metodologia Grading of Recommendations Assessment, Development, and Evaluation (GRADE). En reuniones de trabajo periódicas, el GEG usó la metodología GRADE para revisar la evidencia y formular las recomendaciones, los puntos de buena práctica clínica y el flujograma de manejo. Resultados. La presente GPC abordó 4 preguntas clínicas sobre el tamizaje, diagnóstico, tratamiento de elección y tratamiento adyuvante. En base a estas preguntas se formularon 6 recomendaciones (4 fuertes y 2 condicionales), 19 puntos de buena práctica clínica y 1 flujograma de manejo. Conclusión. El presente artículo resume la metodología y las conclusiones basadas en evidencias de la GPC para el diagnóstico y tratamiento de la retinopatía diabética y el edema macular diabético en EsSalud.

Palabras clave: Retinopatía Diabética; Edema Macular; Guías de Práctica Clínica como Asunto; Enfoque GRADE, Medicina Basada en la Evidencia (fuente: DeCS BIREME).

\section{Abstract}

Introduction. This article summarizes the clinical practice guidelines (CPG) for the diagnosis and treatment of diabetic retinopathy and diabetic macular edema of the Health Social Security of Peru (EsSalud). Objective. To provide clinical recommendations based on evidence for the diagnosis and treatment of diabetic retinopathy and diabetic macular edema in EsSalud. Methods. A guidelines elaborating group (GEG) was conformed by medical specialists and methodologists. The GEG formulated 4 clinical questions intended to be answered by this CPG. For each of these questions systematic searches of systematic reviews and primary studies (when considered pertinent) were carnied out in PubMed during 2018. Evidence was selected in order to reply each of the proposed clinical questions. The certainty of the evidence was evaluated using the Grading of Recommendations Assessment, Development, and Evaluation (GRADE) methodology. During periodic working meetings, the GEG used the GRADE methodology to review the evidence and formulate the recommendations, points of good clinical practice and the management flowchart. Results. The present CPG addressed 4 clinical questions of four topics: screening, diagnosis, treatment of choice and adjuvant treatment. Based on these questions, 6 recommendations (4 strong and 2 conditional), 19 points of good clinical practice, and 1 management flowchart were formulated. Conclusion. This article summarizes the methodology and conclusions based on evidence from the CPG for the diagnosis and treatment of diabetic retinopathy and diabetic macular edema in EsSalud.

Keywords: Diabetic Retinopathy; Macular Edema; Practice Guidelines as Topic; GRADE Approach; Evidence-Based Medicine (source: MeSH NLM). 


\section{INTRODUCCIÓN}

La retinopatía diabética $(\mathrm{RD})$ es una complicación neurovascular de la diabetes mellitus (DM) tipo I y II, que produce ceguera y afecta principalmente a la población laboralmente activa y adulta mayor ${ }^{(1,2,3)}$. Existen dos tipos de RD, la proliferativa (RDP) y no proliferativa (RDNP). Esta última tiende a progresar a la RDP, y se caracteriza por un crecimiento anormal de nuevos vasos sanguíneos en la retina ${ }^{(4)}$. Se estima que más de un tercio de los pacientes con DM tiene algún grado de $\operatorname{RD}^{(5)}$.

Por el daño vascular y consecuente alteración de las barreras hematorretinianas, durante la RD puede existir extravasación de fluido en las capas retinales, y si esto ocurre en el área macular o perimacular, puede producir visión borrosa. A esta condición se le denomina edema macular diabético (EMD), la cual es definida como el engrosamiento de la retina que involucra o está cerca del centro de la mácula (500 micras o menos del centro de la fóvea), y representa la causa más común de pérdida visual en los pacientes con DM ${ }^{(6)}$. El EMD afecta al 3\% de los ojos con RDNP leve, el $38 \%$ de los ojos con RDNP moderado a severo y el $71 \%$ de los ojos con RDP ${ }^{(6,7)}$.

El diagnóstico y tratamiento oportuno de la RD y EMD pueden reducir las complicaciones y discapacidades generadas por estas condiciones. Por este motivo, la Dirección de Guías de Práctica Clínica, Farmacovigilancia y Tecnovigilancia del Instituto de Evaluación de Tecnologías en Salud e Investigación (IETSI) del Seguro Social de Salud del Perú (EsSalud) priorizó la realización de la presente guía de práctica clínica (GPC) con la finalidad de establecer lineamientos basados en evidencias para el diagnóstico y tratamiento de la RD y EMD, cuyos enunciados serán aplicados por profesionales de la salud en EsSalud. El presente artículo es un resumen de dicha GPC.

\section{METODOLOGÍA}

El procedimiento seguido para la elaboración de la presente GPC está detallado en su versión extensa, la cual puede descargarse de la página web del IETSI de EsSalud (http://www.essalud.gob.pe/ ietsi/guias_pract_clini.html).

\section{Conformación del grupo elaborador} de la guía (GEG)

Se conformó un GEG, que incluyó metodólogos y médicos oftalmólogos subespecialistas en retina y vítreo.

\section{Formulación de preguntas}

En concordancia con los objetivos y alcances de esta GPC, el GEG formuló cuatro preguntas clínicas (Tabla 1), cada una de las cuales pudo tener una o más preguntas PICO (Population, Intervention, Comparator, Outcome). A su vez, cada pregunta PICO pudo tener uno o más desenlaces (outcomes) de interés.

\section{Búsqueda y selección de la evidencia}

Para cada pregunta PICO, se realizó la búsqueda y selección de la evidencia. Para ello, durante el 2018 se buscaron revisiones sistemáticas (RS) publicadas como artículos científicos (mediante búsquedas sistemáticas en PubMed y Cochrane Controlled Register of Trials) o realizadas como parte de una GPC previa (mediante una búsqueda sistemática de GPC sobre el tema). Cuando se encontró RS de calidad aceptable, se seleccionó una RS para la toma de decisiones, y se actualizó cuando el GEG lo consideró necesario. Cuando no se encontró ninguna RS de calidad aceptable, se realizó una búsqueda de novo de estudios primarios (Material Suplementario 1).

\section{Evaluación de la certeza de la evidencia}

La certeza de la evidencia para cada desenlace de cada pregunta PICO pudo ser alta, moderada, baja o muy baja (Figura 1). Para evaluar la certeza de la evidencia, se siguió la metodología de Grading of Recommendations Assessment, Development, and Evaluation (GRADE) ${ }^{(8)}$ y se elaboró las tablas de perfiles de evidencias de GRADE (Material Suplementario 2). Finalmente, se le asignó a la pregunta PICO el nivel de certeza más bajo alcanzado por alguno de los desenlaces considerados como críticos.

\section{Formulación de las recomendaciones}

EI GEG revisó la evidencia seleccionada para cada pregunta clínica en reuniones periódicas, y formuló recomendacio- nes fuertes o condicionales (Figura 1) por consenso o por mayoría simple, usando la metodología GRADE ${ }^{(9)}$. Para ello, se tuvo en consideración: 1) Beneficios y daños de las opciones; 2) Valores y preferencias de los pacientes; 3) Aceptabilidad por parte de los profesionales de salud; 4) Factibilidad de las opciones en los establecimientos de salud en EsSalud; y 5) Uso de recursos. Asimismo, el GEG formuló puntos de buenas prácticas clínicas (BPC) en consenso, para los cuales no se realizaron una búsqueda sistemática de la evidencia o no se encontró evidencia suficiente para basar una recomendación; así como enunciados basados en dictámenes de evaluación de tecnología sanitaria.

\section{Revisión por expertos externos}

La presente GPC fue revisada en reuniones con médicos especialistas representantes de otras instituciones $y$ tomadores de decisiones. Asimismo, su versión extensa fue enviada por vía electrónica a expertos externos para su revisión (mencionados en la sección de agradecimientos). El GEG tuvo en cuenta los resultados de estas revisiones para modificar las recomendaciones finales.

\section{Aprobación de la GPC}

La presente GPC fue aprobada para su uso en EsSalud, con Resolución $N^{\circ}$ 95-IETSI-ESSALUD-2019.

\section{Actualización de la GPC}

Esta GPC tiene vigencia de tres años. Al acercarse al fin de este período, se procederá a realizar una revisión rápida de la literatura para decidir si se requiere actualizarla.

\section{RECOMENDACIONES}

La presente GPC abordó cuatro preguntas clínicas con relación a tres temas: diagnóstico, tratamiento de elección y tratamiento adyuvante. En base a dichas preguntas se formularon seis recomendaciones (cuatro fuertes y dos condicionales), 19 puntos de BPC y un flujograma (Tabla 1 y Figura 2).

La presente GPC se enfoca en pacientes mayores de 18 años con diabetes mellitus tipo I o II. En estas personas se 
1

Se definió la certeza de la evidencia, evaluando:

1. Tipo de estudio

2. Riesgo de sesgo

3. Heterogeneidad

4. Evidencia indirecta

5. Imprecisión

6. Sesgo de publicación

7. Tamaño de efecto

8. Relación dosis/respuesta

9. Efecto de confusores

\section{2}

Significado de las categorías de certeza de la evidencia para cada desenlace:

- Alta $(\oplus \oplus \oplus \oplus)$ : Es muy probable que el verdadero efecto sea similar al efecto estimado.

- Moderada $(\bigoplus \oplus \bigoplus \Theta)$ : Es probable que futuros estudios tengan un impacto importante en nuestra confianza en el efecto estimado, y es posible que cambien el estimado.

- Baja $(\oplus \oplus \Theta \Theta)$ : Es muy probable que futuros estudios tengan un impacto importante en nuestra confianza en el efecto estimado, y es probable que cambien el estimado.

- Muy baja $(\oplus \Theta \Theta \Theta)$ : El estimado del efecto no es certero.

Para calificar la certeza general de la pregunta, se toma la peor certeza entre los desenlaces críticos

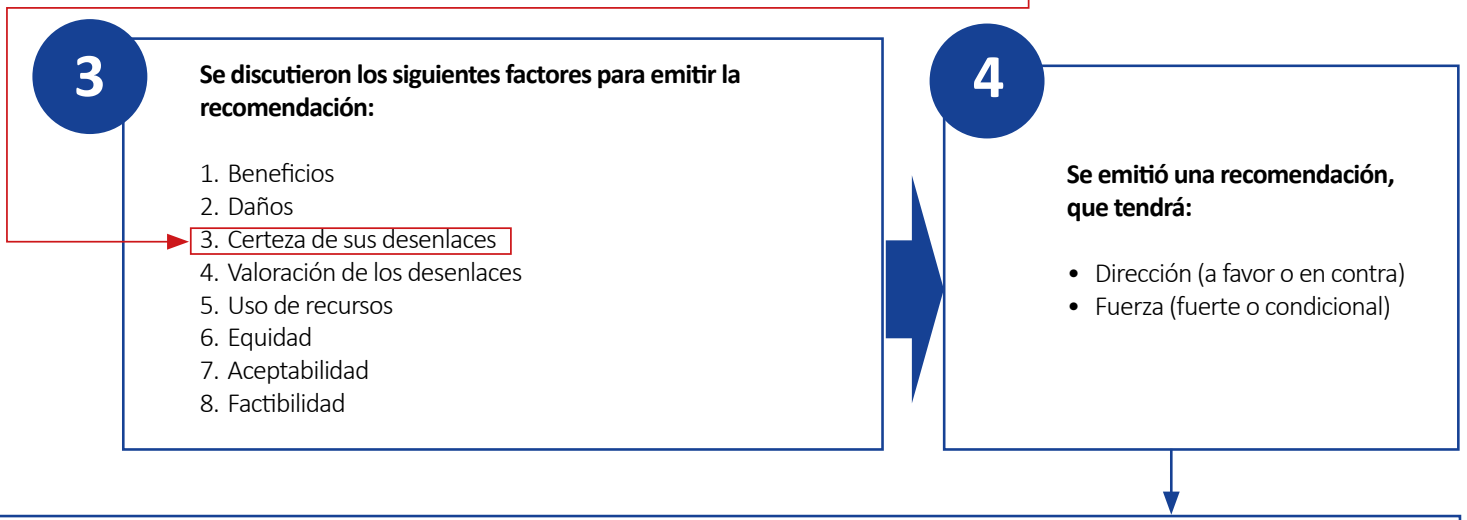

Fuerza de la recomendación:

Fuerte: El GEG considera que todos o la gran mayoría de profesionales que revisan la evidencia disponible seguirán esta recomendación. Se emplea el término "se recomienda".

Condicional: El GEG considera que la mayoría de los profesionales que revisan la evidencia disponible seguirían esta recomendación, pero un grupo no lo seguiría. Se emplea el término "se sugiere".

\section{Dirección de la recomendación:}

A favor: Se recomienda a favor de realizar cierta acción.

En contra: Se recomienda en contra de realizar cierta acción.

Figura 1. Significado de los niveles de certeza de la evidencia y de la fuerza de la recomendación

abordó la realización de la evaluación oftalmológica para el diagnóstico de RD y el uso del OCT para el diagnóstico de EMD, el uso de panfotocoagulación con láser para pacientes con RD, el uso de antiangiogénicos intravítreos en personas con EMD y en personas con RDP que tengan indicación de vitrectomía.

A continuación, se expondrá un resumen del razonamiento seguido para llegar a las recomendaciones y puntos de BPC de cada pregunta clínica.
Pregunta 1: En personas con retinopatía diabética, ¿se debería usar la tomografía de coherencia óptica (OCT) para diagnosticar edema macular diabético (EMD)?

El diagnóstico del EMD es clínico y tradicionalmente la prueba de referencia era la fotografía estereoscópica de fondo de ojo; sin embargo, se considera subjetiva al solo permitir identificar hallazgos sugestivos de edema macular o microaneurismas ${ }^{(10)}$. La OCT podría proporcio- nar una evaluación objetiva y cuantitativa del edema macular, por lo que cada vez más es reconocida como el método de diagnóstico de referencia para EMD ${ }^{(11)}$.

Para esta pregunta se encontró una sola RS: Virgili $2015^{(11)}$, que presentó una adecuada calidad metodológica (AMSTAR-2: 14/16). Esta RS realizó un metaanálisis (MA) de 9 estudios observacionales (1303 ojos, 759 personas) que tuvo como objetivo determinar la precisión diagnóstica de la OCT para detectar el 
Tabla 1. Recomendaciones en base a preguntas clínicas con relación al diagnóstico, tratamiento de elección y tratamiento adyuvante de la retinopatía diabética y edema macular diabético

\begin{tabular}{|c|c|c|c|}
\hline № & Enunciado & Tipo * & Certeza ** \\
\hline \multicolumn{4}{|c|}{ Diagnóstico } \\
\hline \multicolumn{4}{|c|}{$\begin{array}{l}\text { Pregunta 1: En personas con retinopatía diabética, ¿se debería usar la tomografía de coherencia óptica (OCT) para diagnosticar edema macular } \\
\text { diabético (EMD)? }\end{array}$} \\
\hline 1.1 & $\begin{array}{l}\text { En personas con diabetes mellitus (DM), el diagnóstico de retinopatía diabética } \\
\text { (RD) es clínico, mediante el examen de fondo de ojo dilatado por el médico } \\
\text { oftalmólogo, sea en lámpara de hendidura con lupa 78D, 90D o por oftalmoscopía } \\
\text { indirecta. }\end{array}$ & BPC & \\
\hline 1.2 & $\begin{array}{l}\text { En personas con DM, la evaluación oftalmológica realizada por un médico } \\
\text { oftalmólogo debe incluir la anamnesis, evaluación de la agudeza visual, presión } \\
\text { intraocular y la evaluación del segmento anterior que incluya la búsqueda de } \\
\text { neovascularización en iris y ángulo; y examen de fondo de ojo con dilatación } \\
\text { pupilar. }\end{array}$ & BPC & \\
\hline
\end{tabular}

En personas con DM, considerar realizar la evaluación oftalmológica inicial y de control según el siguiente esquema:

Evaluaciones oftalmológicas en personas con Diabetes Mellitust

Indicación

Tipo de diabetes

Acción

1.3
Al momento del

DM1 diagnóstico

Evaluación

\begin{tabular}{cc} 
DM1 & $\begin{array}{c}\text { Al momento del } \\
\text { diagnóstico }\end{array}$ \\
\hline DM2 & $\begin{array}{c}\text { Al momento del } \\
\text { diagnóstico }\end{array}$ \\
\hline
\end{tabular}

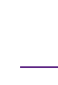

oftalmológica inicia

Gestación en paciente
con DM1 o DM2

\begin{tabular}{lcc}
$\begin{array}{l}\text { Evaluación } \\
\text { oftalmológica de } \\
\text { control }\end{array}$ & DM1 & $\begin{array}{c}\text { Anual a partir del quinto } \\
\text { año de la evaluación } \\
\text { oftalmológica inicial }\end{array}$ \\
\cline { 2 - 3 } & DM2 & Anual \\
\hline
\end{tabular}

† Modificado de: ADA Standards of Medical Care in Diabetes- 2018
Antes y por lo menos

durante el primer trimestre de gestación Anual
BPC

En personas con DM, considerar clasificar la retinopatía diabética según la Clasificación Internacional de Retinopatía Diabética.

\section{Clasificación Internacional de Retinopatía Diabéticał}

\section{Retinopatía Diabética \\ Hallazgos observables con oftalmoscopía} dilatada

\begin{tabular}{ll} 
RD no aparente & No hay anomalías \\
\hline RDNP leve & Solamente microaneurismas \\
\hline RDNP moderada & $\begin{array}{l}\text { Microaneurismas y otros signos (por ejemplo, } \\
\text { hemorragias en manchas, exudados duros, } \\
\text { manchas algodonosas), pero menos que en RDNP }\end{array}$
\end{tabular}

severa

\begin{tabular}{ll}
\hline RDNP severa & RDNP moderada con cualquiera de los siguientes: \\
& - Hemorragias intrarretinianas ( $\geq 20$ en cada \\
& cuadrante) \\
& - Rosarios venosos definidos (en 2 cuadrantes) \\
& - Anomalías microvasculares intrarretinianas (en \\
& 1 cuadrante) \\
& - Sin signos de retinopatía proliferativa \\
\hline RD proliferativa & RDNP severa y 1 o más de los siguientes: \\
& - Neovascularización \\
& - Hemorragia vítrea/preretiniana \\
\hline
\end{tabular}

† Fuente: ICO Guidelines for Diabetic Eye Care 2018 
Tabla 1 (continuación). Recomendaciones en base a preguntas clínicas con relación al diagnóstico, tratamiento de elección y tratamiento adyuvante de la retinopatía diabética y edema macular diabético

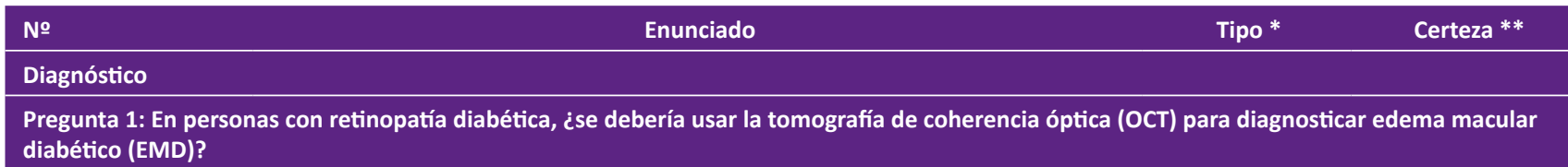
diabético (EMD)?

1.5

1.6

1.7

1.8

En personas con DM, se debe optimizar el control metabólico de la glucosa y de los factores de riesgo como presión arterial y lípidos séricos para reducir el riesgo o disminuir la progresión de la RD.

BPC

En personas con DM, informar que la RD es una complicación crónica y progresiva pero un adecuado autocontrol metabólico (glucosa, presión arterial y lípidos), evaluación oftalmológica temprana y tratamiento oportuno pueden disminuir el riesgo de ceguera.

En personas con RD, se sospecha de EMD cuando el paciente presenta engrosamiento de la retina y/o presencia de exudados duros en área macular o perimacular como hallazgo en el fondo de ojo con dilatación pupilar realizado por el médico oftalmólogo, sea en lámpara de hendidura con lupa 78D o 90D, o por oftalmoscopía indirecta.

En personas con RD y sospecha de EMD, recomendamos utilizar OCT para diagnosticar y clasificar el EMD.

$\mathrm{BPC}$

En personas con RD, considerar clasificar el EMD según la Clasificación Internacional de Edema Macular Diabético.

Clasificación Internacional de Edema Macular Diabéticoł

\section{Edema Macular \\ Diabético}

Hallazgos observables con oftalmoscopía

\section{dilatada}

Sin engrosamiento de retina o exudados duros en la mácula

1.9

Sin EMD

EMD sin compromiso
central

Engrosamiento de la retina en la mácula sin compromiso de la zona subcampo central que es de $1 \mathrm{~mm}$ de diámetro.

EMD con compromiso
central

Engrosamiento de la retina en la mácula con compromiso de la zona subcampo central que es de $1 \mathrm{~mm}$ de diámetro.

† Fuente: ICO Guidelines for Diabetic Eye Care 2018

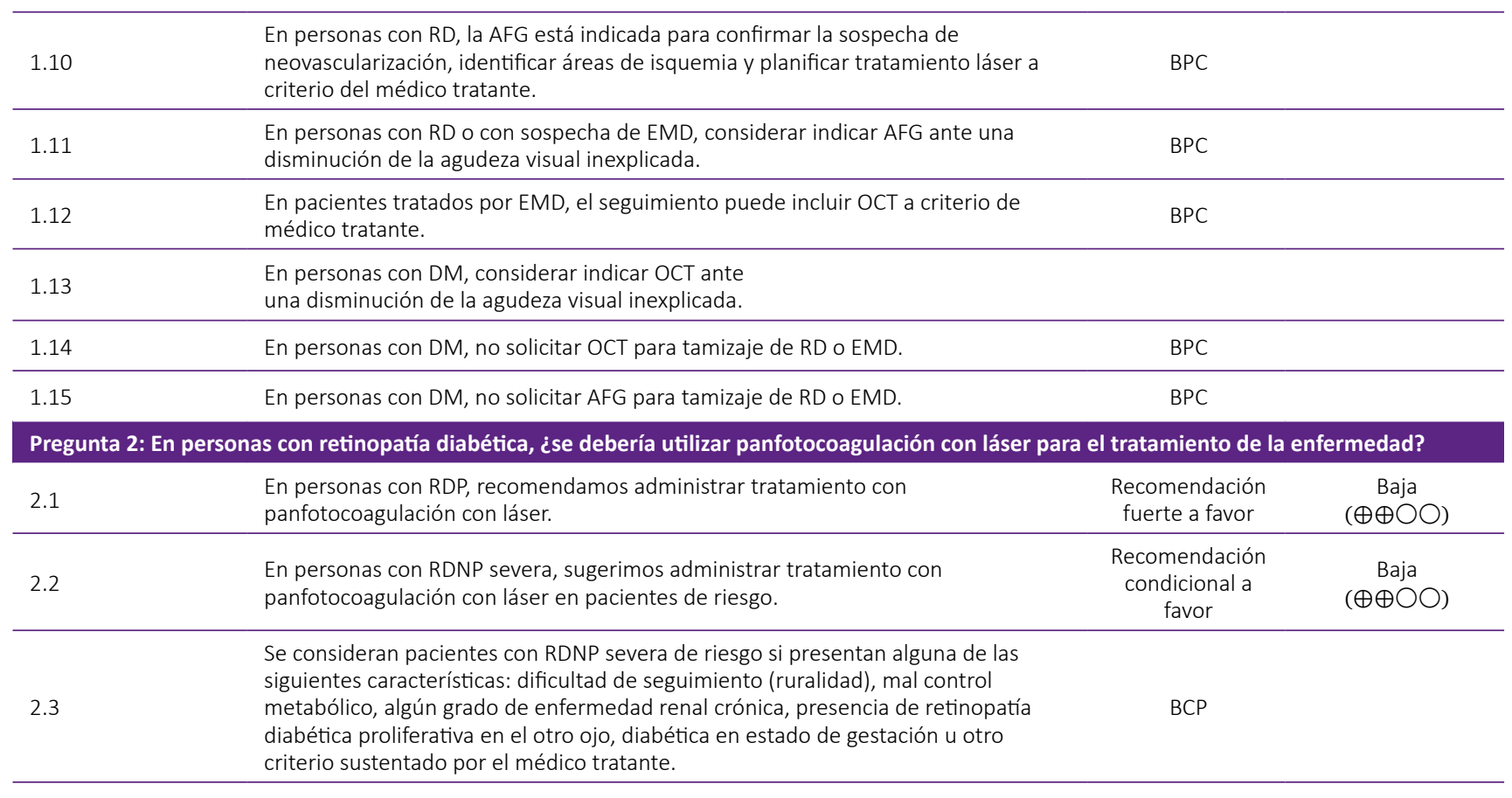


Tabla 1 (continuación). Recomendaciones en base a preguntas clínicas con relación al diagnóstico, tratamiento de elección y tratamiento adyuvante de la retinopatía diabética y edema macular diabético

\begin{tabular}{|lcc}
\hline № & Enunciado & Tipo * Certeza ** \\
\hline Diagnóstico & & \\
\hline Pregunta 2: En personas con retinopatía diabética, ¿̇se debería utilizar panfotocoagulación con láser para el tratamiento de la enfermedad?
\end{tabular}

En personas con RD, la frecuencia con la que se realizará las evaluaciones oftalmológicas de seguimiento será definida por la severidad, presencia de signos de neovascularización u otros criterios sustentados por el médico tratante; considerando intervalos de tiempo según el siguiente esquema:

\begin{tabular}{|c|c|c|}
\hline \multirow{6}{*}{2.4} & \multicolumn{2}{|c|}{$\begin{array}{c}\text { Evaluaciones oftalmológicas de control en personas con Retinopatía } \\
\text { Diabéticat }\end{array}$} \\
\hline & Retinopatía Diabética & Periodicidad de control \\
\hline & RDNP leve & 6 a 12 meses \\
\hline & RDNP moderada & 3 a 6 meses \\
\hline & RDNP severa & $<3$ meses \\
\hline & RDP & $<1$ mes \\
\hline
\end{tabular}

Pregunta 3: En personas con edema macular diabético, ¿se debería utilizar antiangiogénicos intravítreos para el tratamiento de la enfermedad?

\begin{tabular}{llll}
3.1 & $\begin{array}{l}\text { En personas con EMD con compromiso central y AV de 20/30 o peor, recomendamos } \\
\text { administrar bevacizumab intravítreo como tratamiento de primera línea. }\end{array}$ & $\begin{array}{c}\text { Recomendación } \\
\text { fuerte a favor }\end{array}$ & $\begin{array}{c}\text { Moderada } \\
(\oplus \oplus \oplus \bigcirc)\end{array}$ \\
\hline 3.2 & $\begin{array}{l}\text { En personas con EMD con compromiso central, recomendamos no administrar } \\
\text { corticoides intravítreos como tratamiento de primera línea. }\end{array}$ & $\begin{array}{c}\text { Recomendación } \\
\text { fuerte en contra }\end{array}$ & Baja \\
& & $\oplus \oplus \bigcirc)$ &
\end{tabular}

En personas con EMD sin compromiso central o AV mejor que 20/30, el
3.3 tratamiento puede incluir seguimiento cercano, fotocoagulación con láser o bevacizumab intravítreo a criterio del médico tratante.

Con respecto al uso de bevacizumab fuera de la etiqueta:

3.4

- Realizar el consentimiento informado antes de su aplicación.

- Aplicar 1.25 mg en $0.05 \mathrm{ml}$ por inyección intravítrea.

- Seguir el protocolo de preparación y administración elaborado por IETSI EsSalud

3.5

En personas con EMD con evidencia de tracción vitreomacular y/o membrana epirretiniana mediante OCT, considerar realizar tratamiento con vitrectomía.
BPC

BPC

BPC

Pregunta 4: En personas con retinopatía diabética proliferativa con indicación de vitrectomía, ¿se debería utilizar antiangiogénicos intravítreos como tratamiento adyuvante a la vitrectomía?

En pacientes con RDP con indicación de vitrectomía, sugerimos administrar bevacizumab intravítreo preoperatorio entre 3 a 7 días previos a la vitrectomía, considerando el riesgo de desprendimiento de retina traccional después de la inyección de antiangiogénico en dichos pacientes.

$\begin{array}{lc}\begin{array}{c}\text { Recomendación } \\ \text { condicional a } \\ \text { favor }\end{array} & \text { Baja } \\ & (\oplus \oplus \bigcirc \bigcirc)\end{array}$

favor

* Recomendación (R) o punto de buena práctica clínica (BPC)
** La fuerza, dirección y calidad de la evidencia sólo se establecen para las recomendaciones, más no para los puntos de BPC.

DM: Diabetes mellitus; RD: Retinopatía diabética; RDNP: Retinopatía diabética no proliferativa; RDP: Retinopatía diabética proliferativa; EMD: Edema macular diabético; OCT: Tomografía de coherencia óptica; AFG: Angiofluoresceinografía retinal; ADA: American Association Ophthalmology; ICO: International Council of Ophthalmology

EMD en personas con RD, considerando como prueba de referencia la evaluación de fondo de ojo (en tres estudios se empleó biomicroscopia, en cuatro estudios se empleó fotografía estereoscópica y en dos estudios se empleó ambas técnicas). Esta RS encontró que la OCT tuvo una sensibilidad de 81\% (IC 95\%: 74\% a 86\%) y una especificidad de $85 \%$ (IC 95\%: 75\% a $91 \%)$. Sin embargo, no se puede determinar con certeza la precisión diagnósti- ca de la OCT al no existir una prueba de referencia (gold standard) con el cual comparar.

En base a lo evidenciado, el GEG consideró que en la actualidad la OCT es la prueba de referencia para diagnosticar EMD, que además permite realizar el seguimiento en los pacientes con tratamiento. Por este motivo, se decidió emitir una recomendación a favor del uso de la OCT para diagnosticar y clasificar EMD en personas con RD. A pesar de que la certeza de la evidencia fue baja, se consideró que no usar OCT conllevaría dificultades en el diagnóstico y seguimiento de EMD, por lo cual se decidió emitir una recomendación fuerte.

Puntos de BPC: Se estableció 14 puntos de BPC sobre: el diagnóstico y la evaluación oftalmológica de pacientes con RD según lo recomendado por la American Academy of Ophthalmology (AAO) ${ }^{(12)}$; la 


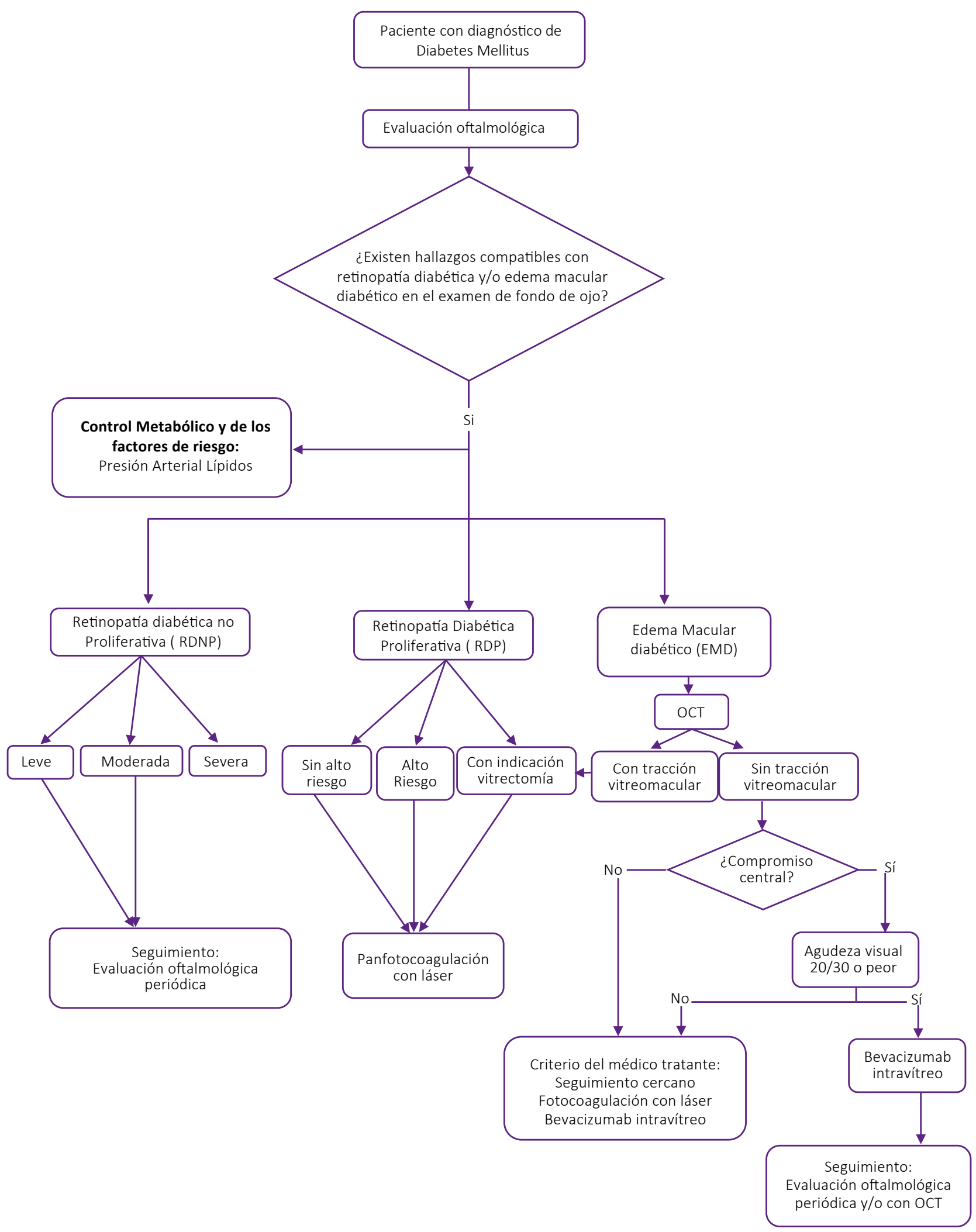

Nota: No se emitieron recomendaciones para pacientes con edema macular persistente o refrectario a tratamiento con antiangiogénicos intravitreos.

OTC: Tomografía por coherencia óptica

Figura 2. Flujograma del manejo de la retinopatía diabética y edema macular diabético 
evaluación oftalmológica inicial en pacientes con diabetes según lo recomendado por la ADA ${ }^{(1)}$; el uso de la clasificación internacional de RD según la International Council of Ophthalmology (ICO) (4); el control de los factores de riesgo asociados a la RD y el autocontrol metabólico según lo recomendado de la $\mathrm{ADA}^{(1)}$; el empleo de la clasificación internacional de EMD según la ICO; el uso de angiofluorescencia retinal; el seguimiento de los pacientes con EMD; y el adecuado uso de la OCT en pacientes con RD según las recomendaciones de la AAO ${ }^{(12)}$.

Pregunta 2: En personas con retinopatía diabética, ¿se debería utilizar panfotocoagulación con láser para el tratamiento de la enfermedad?

La panfotocoagulación con láser es una técnica que destruye selectivamente la retina hipóxica, lo cual disminuye la demanda de oxígeno, citoquinas y factores de crecimiento (como el factor de crecimiento endotelial vascular), disminuyendo así la neovascularización en personas con $\operatorname{RD}^{(13,14)}$.

Para responder esta pregunta se encontró una sola RS: Evans $2014{ }^{(15)}$, que presentó una adecuada calidad metodológica (AMSTAR-2: 14/16). Esta RS incluyó ensayos clínicos aleatorizados (ECA) realizados en pacientes con retinopatía diabética proliferativa (RDP) y con retinopatía diabética no proliferativa (RDNP).

Esta RS encontró que al año de seguimiento, el grupo que recibió panfotocoagulación con láser tuvo similar agudeza visual (definida como la pérdida de 15 letras o más en la mejor agudeza visual corregida [MAVC] (11,5\% versus 11,6\%, RR: 0,99; IC 95\%: 0,89 - 1,11), menor riesgo de desarrollar pérdida severa de la visión (MAVC < 6/60) (2,5\% versus 4,4\%, RR: 0,46; IC 95\%: 0,24 - 0,86) y menor progresión de la retinopatía diabética (23,1\% versus 41,0\%, RR: 0,49; IC 95\%: $0,37-0,64)$; en comparación con el grupo control. No se mostró datos sobre pérdida de visión periférica, un efecto adverso posible de la panfotocoagulación.

Se observa que la panfotocoagulación con láser presenta beneficios significativos, por lo cual se decidió emitir recomendaciones a favor de su uso en pacientes con RDP y RDNP. Respecto a la fuerza, se decidió emitir una recomendación fuerte para pacientes con RDP (quienes, al presentar mayor riesgo de progresión de la enfermedad, se verían más beneficiados por la intervención). En tanto que se decidió emitir una recomendación condicional en pacientes con RDNP (quienes, al presentar menor riesgo de progresión de la enfermedad, se verían menos beneficiados por la intervención).

Puntos de BPC: Se estableció dos puntos de BPC sobre: quienes son los pacientes con RDNP en riesgo para nuestro contexto en opinión del GEG; y la frecuencia de las evaluaciones oftalmológicas en pacientes con RD según lo recomendado por la AAO ${ }^{(12)}$.

Pregunta 3: En personas con edema macular diabético, ¿se debería utilizar antiangiogénicos intravítreos para el tratamiento de la enfermedad?

El EMD se caracteriza por el incremento de la permeabilidad vascular y la consecuente acumulación de líquido exudativo en la mácula, lo que produce edema macular ${ }^{(16)}$. Esto tiene como mediador clave al factor de crecimiento endotelial vascular (VEGF), por lo que se ha sugerido que las inyecciones intravítreas de medicamentos inhibidores del VEGF serían útiles en el tratamiento del EMD ${ }^{(17)}$. Para esta pregunta clínica se realizaron tres preguntas PICO:

La primera pregunta PICO comparó los anti-VEGF intravítreos vs fotocoagulación con láser. Para responder esta pregunta PICO se encontró solo una RS: Virgili $2018^{(17)}$, que presentó una adecuada calidad metodológica (AMSTAR-2: 15/16). Esta RS realizó un meta-análisis en red (MAR) y en la comparación mixta encontró que el grupo que recibió antiVEGF (bevacizumab intravítreo) presentó mayor probabilidad de "ganancia igual o mayor a tres líneas de agudeza visual" al año (RR: 2,47; IC 95\%: 1,81-3,37), mayor agudeza visual al año (diferencia de medias [DM]:-0,12; IC 95\%:-0.15 a-0.09), menor volumen macular al año (DM:-46 um; IC 95\%:-78 a-14), y similar incidencia de efectos adversos a los dos años (RR: 0,93; IC 95\%: 0,73 - 1,19) en comparación con el grupo que recibió fotocoagulación con láser.
La segunda pregunta PICO comparó los anti-VEGF intravítreos entre sí: Para esta pregunta PICO se encontró una sola RS: Virgili $2018^{(17)}$, que presentó una adecuada calidad metodológica (AMSTAR-2: 15/16). Esta RS en la comparación mixta encontró que, a un año de seguimiento, el grupo que recibió bevacizumab intravítreo tuvo similar "ganancia mayor o igual a tres líneas de agudeza visual" (RR: 1,11; IC 95\%: 0,87-1,43), similar agudeza visual (DM: 0,00; IC 95\%:-0,02 a 0,03), mayor volumen macular (DM: -29 $\mu \mathrm{m}$; IC 95\%:-58 a-1), y similar incidencia de efectos adversos (mortalidad por todas las causas, eventos aterotrombóticos) (RR: 1,04; IC 95\%: 0,84- 1,28) en comparación al grupo que recibió ranibizumab intravítreo.

Se observa que el empleo de anti-VEGF intravítreo presenta mayores beneficios $y$ menores daños en comparación con fotocoagulación con láser. Además, entre los anti-VEGF, el bevacizumab intravítreo presenta similares beneficios y efectos adversos que el ranibizumab intravítreo a un menor costo. Por ello, se decidió emitir una recomendación a favor del uso de bevacizumab como tratamiento de primera línea. La certeza de la evidencia fue moderada, por lo cual se decidió emitir una recomendación fuerte.

La tercera pregunta PICO comparó la combinación de anti-VEGF con corticoide intravítreo vs anti-VEGF. Para esta pregunta PICO se encontró una sola RS: Mehta $2018^{(16)}$, que presentó una adecuada calidad metodológica (AMSTAR-2: 14/16). Esta RS incluyó en la mayoría de ECA al bevacizumab 1,25 mg/0,05 ml (7/8) como anti- VEGF y como inyección de corticoide intravítreo a la triamcinolona 2 mg/0,05 ml (6/8). Esta RS encontró que a un año de seguimiento, el grupo tratado con anti-VEGF y corticoide intravítreos presentó similar agudeza visual (DM:-2,29; IC 95\%:-6,03 a 1,45), similar volumen macular (DM:-0,20 $\mu \mathrm{m}$; IC 95\%: $-37,14$ - 37,53), y mayor incidencia de eventos adversos (29,3\% versus $7,7 \%$; OR: 5,66; IC 95\%: 3,62-8,84) en comparación al grupo al que solo se le administró anti-VEGF.

Considerando que la combinación de bevacizumab con corticoide intravítreos 
no presentó más beneficios, pero sí más efectos adversos respecto al uso de solo bevacizumab, se decidió emitir una recomendación en contra del empleo de esta combinación. A pesar de que la certeza de la evidencia fue baja, se consideró que su indicación generaría un mayor uso de recursos, por lo cual se decidió emitir una recomendación fuerte.

Puntos de BPC: Se establecieron tres puntos de BPC sobre: el tratamiento en pacientes con EMD sin compromiso central o agudeza visual mejor que 20/30 según lo recomendado por la ICO (4); el uso del bevacizumab fuera de la etiqueta según las "Recomendaciones para la preparación de bevacizumab inyección intravítrea, IETSI-EsSalud, 2018"; y la indicación de vitrectomía en pacientes con EMD con evidencia de tracción vitreomacular y/o membrana epirretiniana mediante OCT según la ICO.

Pregunta 4: En personas con retinopatía diabética proliferativa con indicación de vitrectomía, ¿se debería utilizar antiangiogénicos intravítreos como tratamiento adyuvante a la vitrectomía?

\section{De la evidencia a la decisión:}

La vitrectomía pars plana es un procedimiento quirúrgico usado para el tratamiento de complicaciones graves de la RDP, como el desprendimiento de retina traccional y la hemorragia vítrea ${ }^{(18)}$. La complicación más importante posterior a una vitrectomía es la hemorragia de la cavidad vítrea posterior (POVCH), que ocurre en un $30 \%$ de los casos.

La POVCH se puede presentar en un periodo temprano (primeros días del postoperatorio) o tardío (meses después de la cirugía), retrasa la recuperación visual y conduce a una elevada presión intraocular, esto dificulta aún más el tratamiento de la RD. El 10\% de los pacientes requieren cirugía de revisión, lo que tiene implicancias significativas para los recursos como el tiempo y el costo ${ }^{(22)}$.

El VEGF aumenta la permeabilidad vascular y produce neovascularización en la cavidad vítrea posterior ${ }^{(19)}$, por lo cual se ha propuesto que el uso de anti-VEGF (preoperatorio a la vitrectomía) puede reducir la incidencia de $\mathrm{POVCH}{ }^{(20)}$.
Para responder esta pregunta se encontró dos RS: Zhao $2018^{(21)}$ y Smith $2015^{(22)}$, que presentaron una adecuada calidad metodológica (AMSTAR-2: 9/16 y 14/16; respectivamente). En estas RS la mayoría de estudios incluidos usó bevacizumab, y el tiempo transcurrido entre la administración de anti-VEGF intravítreo y la vitrectomía varió de 1 a 21 días, la mayoría reportó que la administración de anti-VEGF fue de 7 días antes de la vitrectomía (9/15).

La RS de Smith ${ }^{(22)}$ evaluó la mayoría de los desenlaces relevantes, excepto agudeza visual, la cual fue evaluada por la RS de Zhao ${ }^{(21)}$. La RS de Smith ${ }^{(22)}$ realizó MA de 10 ECAs y encontró que el grupo tratado preoperatoriamente con anti-VEGF intravítreo presentó menor incidencia de $\mathrm{POVCH}$ temprano (cuatro semanas) (9,05\% versus $31,1 \%$, RR: 0,35; IC 95\%: 0,23-0,53), similar incidencia de POVCH tardío (seis meses) (13,04\% versus 19,75\%, RR: 0,72; IC 95\%: 0,30-1,72), menor incidencia de lavado de la cavidad vítrea (6 meses) (1,91\% versus 10,44\%, RR: 0,19; IC 95\%: 0,06 a 0,67), y similar incidencia de eventos adversos (desprendimiento de retina) (3,36\% versus $9,14 \%$, RR: 0,46; IC 95\%: 0,19 a 1,08) en comparación con el grupo control.

La RS de Zhao $2018^{(21)}$ realizó un MA de siete ECAs y encontró que el grupo tratado preoperatoriamente con antiVEGF intravítreo presentó mayor agudeza visual en comparación con el grupo control (DM:-0,30; IC 95\%:-0,44 a-0,16).

En general se observa que el grupo que usa inyección intravítrea preoperatoria de anti-VEGF (bevacizumab) presentó menor riesgo de $\mathrm{POVH}$ y mejor agudeza visual, por lo que se decidió emitir una recomendación a favor de su uso. Además, considerando que la certeza de la evidencia fue baja, por lo que se decidió emitir una recomendación condicional.

\section{AGRADECIMIENTOS}

A Fiorella Salvador Salvador (Instituto Mexicano de Oftalmología) y Renata del Carmen García Franco (Instituto Mexicano de Oftalmología) por sus aportes durante la revisión de la presente guía de práctica clínica.

\section{REFERENCIAS BIBLIOGRÁFICAS}

1. Solomon SD, Chew E, Duh EJ, Sobrin L, Sun JK, VanderBeek BL, et al. Diabetic Retinopathy: A Position Statement by the American Diabetes Association. Diabetes care. 2017;40(3):412-8. DOI: 10.2337/ dc16-2641

2. Antonetti DA, Klein R, Gardner TW. Diabetic retinopathy. The New England journal of medicine. 2012;366(13):1227-39. DOI: 10.1056/NEJMra1005073

3. Cheung N, Mitchell P, Wong TY. Diabetic retinopathy. Lancet (London, England). 2010;376(9735):124-36. DOI: 10.1016/S0140-6736(09)62124-3

4. Wong TY, Sun J, Kawasaki R, Ruamviboonsuk P, Gupta N, Lansingh VC, et al. Guidelines on Diabetic Eye Care: The International Council of Ophthalmology Recommendations for Screening, Follow-up, Referral, and Treatment Based on Resource Settings. Ophthalmology. 2018;125(10):1608-22. DOI: 10.1016/j.ophtha.2018.04.007

5. Lee R, Wong TY, Sabanayagam C. Epidemiology of diabetic retinopathy, diabetic macular edema and related vision loss. Eye and vision (London, England). 2015;2:17. DOI: 10.1186/s40662-0150026-2

6. Bandello F, Battaglia Parodi M, Lanzetta P, Loewenstein A, Massin P, Menchini F, et al. Diabetic Macular Edema. Developments in ophthalmology. 2017;58:102-38. DOI: 10.1159/000320075

7. Bandello F, Battaglia Parodi M, Lanzetta P, Loewenstein A, Massin P, Menchini F, et al. Diabetic macular edema. Developments in ophthalmology. 2010;47:73-110. DOI: https://doi. org/10.1159/000320075

8. Balshem $H$, Helfand $M$, Schünemann $H J$, Oxman AD, Kunz R, Brozek J, et al. GRADE guidelines: 3. Rating the quality of evidence. Journal of clinical epidemiology. 2011;64(4):401-6. DOI: 10.1016/j. jclinepi.2010.07.015

9. Andrews J, Guyatt G, Oxman AD, Alderson P, Dahm P, Falck-Ytter Y, et al. GRADE guidelines: 14. Going from evidence to recommendations: the significance and presentation of recommendations. Journal of clinical epidemiology. 2013;66(7):719-25. DOI: 10.1016/j.jclinepi.2012.03.013

10. Photocoagulation for diabetic macular edema. Early Treatment Diabetic Retinopathy Study report number 1. Early Treatment Diabetic Retinopathy Study research group. Archives of ophthalmology (Chicago, III : 1960). 1985;103(12):1796-806.

11. Virgili G, Menchini F, Casazza G, Hogg R, Das RR, Wang $X$, et al. Optical coherence tomography (OCT) for detection of macular oedema in patients with diabetic retinopathy. The Cochrane database of systematic reviews. 2015;1:Cd008081. DOI: 10.1002/14651858.CD008081.pub3

12. American Academy of Ophthalmology Retina/ Vitreous Panel. Preferred Practice Pattern $®$ Guidelines. Diabetic Retinopathy. San Francisco, CA: American Academy of Ophthalmology; 2017. Disponible en: www.aao.org/ppp.

13. Aiello LP, Sun W, Das A, Gangaputra S, Kiss S, Klein R, et al. Intensive diabetes therapy and ocular surgery in type 1 diabetes. The New England journal of medicine. 2015;372(18):1722-33. DOI: 10.1056/ NEJMoa1409463

14. BMJP G. Tight blood pressure control and risk of macrovascular and microvascular complications in type 2 diabetes: UKPDS 38. BMJ. 1998;317(7160):703-13 
15. Evans JR, Michelessi M, Virgili G. Laser photocoagulation for proliferative diabetic retinopathy. The Cochrane database of systematic reviews. 2014(11):Cd011234. DOI: 10.1002/14651858. CD011234.pub2

16. Mehta H, Hennings C, Gillies MC, Nguyen V Campain A, Fraser-Bell S. Anti-vascular endothelia growth factor combined with intravitreal steroids for diabetic macular oedema. The Cochrane database of systematic reviews. 2018;4:Cd011599. DOI: 10.1002/14651858.CD011599.pub2

17. Virgili G, Parravano M, Evans JR, Gordon I, Lucenteforte E. Anti-vascular endothelial growth factor for diabetic macular oedema: a network meta-analysis. The Cochrane database of systematic reviews.
2017;6:Cd007419. DOI: 10.1002/14651858. CD007419.pub5

18. Oellers P MT. Surgery for proliferative diabetic retinopathy: new tips and tricks. J Ophthalmic Vis Res. 2016;11(1):93-9. DOI: 10.4103/2008-322X.180697

19. Yau GL, Silva PS, Arrigg PG, Sun JK. Postoperative Complications of Pars Plana Vitrectomy for Diabetic Retinal Disease. Seminars in ophthalmology. 2018;33(1):126-33. DOI: 10.1080/08820538.2017.1353832

20. Morera Y, Gonzalez R, Lamdan H, Perez L, Gonzalez $Y$, Aguero J, et al. Vaccination with a mutated variant of human Vascular Endothelial Growth Factor (VEGF) blocks VEGF-induced retinal neovascularization in a rabbit experimental model. Experimental eye research. 2014;122:102-9. DOI: 10.1016/j. exer.2014.03.006

21. Zhao XY, Xia S, Chen YX. Antivascular endothelial growth factor agents pretreatment before vitrectomy for complicated proliferative diabetic retinopathy: a meta-analysis of randomised controlled trials. The British journal of ophthalmology. 2018;102(8):107785. DOI: 10.1136/bjophthalmol-2017-311344

22. Smith JM, Steel DH. Anti-vascular endothelial growth factor for prevention of postoperative vitreous cavity haemorrhage after vitrectomy for proliferative diabetic retinopathy. The Cochrane database of systematic reviews. 2015(8):Cd008214. DOI: 10.1002/14651858.CD008214.pub3 